\title{
Dissecting Aneurysm of the Recurrent Artery of Heubner in a Patient With Osteogenesis Imperfecta
}

\author{
Kevin Mansfield, Ralph Rahme
}

Keywords: Dissection, intracranial aneurysm, osteogenesis imperfecta, recurrent artery of Heubner, subarachnoid hemorrhage

doi:10.1017/cjn.2015.295

Can J Neurol Sci. 2015; 42: 461-465

Spontaneous intracranial dissections are a rare cause of subarachnoid hemorrhage (SAH), accounting for $2 \%$ to $3 \%$ of cases. ${ }^{1,2}$ Dissecting aneurysms most commonly affect the large proximal intracranial vessels, although small distal cerebral vessels may rarely be involved. ${ }^{1-4}$ However, to the best of our knowledge, dissecting aneurysms of the recurrent artery of Heubner (RAH), a large named perforator arising from the anterior cerebral artery (ACA), have not been previously observed. We present an exceptional case of ruptured dissecting aneurysm of the RAH in a patient with osteogenesis imperfecta (OI), an inherited disorder of collagen synthesis.

\section{CASE Report}

A 50-year-old woman was transferred to us for evaluation and management of spontaneous SAH and intraventricular hemorrhage. She had been found unresponsive by her family and was initially seen in an outside hospital, where she was found to have a Glasgow coma score of 5 (decorticate posturing) and was thus intubated. Head computed tomography (CT) scan revealed diffuse cisternal SAH predominating in the anterior interhemispheric fissure and tetraventricular hemorrhage with hydrocephalus (Figure 1A,B). A right frontal external ventricular drain was placed emergently, revealing an opening pressure of $20 \mathrm{~cm}$ of water. Following cerebrospinal fluid diversion, her neurological examination improved slightly, measured by her withdrawing all four extremities to pain.

CT angiography was obtained, demonstrating a small fusiform aneurysmal dilation of the well-developed left RAH (Figure 1C). Cerebral angiography confirmed the presence of a $4 \mathrm{~mm} \times 1.5 \mathrm{~mm}$ fusiform aneurysm arising from the proximal portion of the left RAH overlying the A1 segment of the left ACA (Figure 1D). The A1 vessel was mildly deformed, suggesting that the aneurysm was actually larger in size and had a thrombosed, nonangiographically visible portion (Figure 1D). Given the "pearl-on-string" appearance of the aneurysm and its atypical location, a dissecting nature was strongly suspected. Moreover, there was angiographic evidence of an old healed dissection of the right internal carotid artery, just proximal to its entrance into the carotid canal, and occlusion of the proximal right vertebral artery at its V1-V2 junction, just proximal to its entrance into the right $\mathrm{C} 6$ foramen transversarium, also suggesting an old dissection (Figure 1E,F). Consideration was given for obtaining a brain magnetic resonance imaging to demonstrate the intramural thrombus and confirm the diagnosis of intracranial dissection. However, given the patient's critical condition and the expectation that magnetic resonance imaging findings would not drastically change our management strategy, this study was not performed in order to avoid delaying definitive treatment.

Further questioning of the family revealed that the patient had type I (autosomal dominant) OI, as did roughly 50\% of her siblings and children. Interestingly, the patient's mother had died of aneurysmal SAH and one of her maternal aunts had had surgical clip ligation of an intracranial aneurysm. The patient and one of her daughters exhibited obvious clinical signs of OI, including blue-tinted sclerae, joint hyperlaxity, and finger deformities secondary to multiple prior healed fractures (Figure 2). The patient never smoked tobacco and had no history of hypertension.

Given the location and configuration of the aneurysm, endovascular embolization was not felt to be a good treatment option. In fact, given the dissecting fusiform nature of the aneurysm, preservation of the RAH would have been virtually impossible by endovascular means. Although, not infrequently, RAH occlusion may only cause clinically silent infarctions, severe neurological deficits have nonetheless been observed in this setting. ${ }^{5}$ Likewise, the small size of this vessel and its sharp angle of takeoff from the ACA would have made superselective catheterization very technically challenging and increased the risk of microwire perforation and intraprocedural rupture. Thus, the decision was made to surgically explore the aneurysm and attempt clip reconstruction of the RAH. Under continuous intraoperative neurophysiological monitoring (motor-evoked potentials), the aneurysm was exposed via a standard left pterional transsylvian approach. The aneurysm had a fusiform, pearl-on-string appearance and was much larger than its angiographically visible portion. There was an obvious intramural thrombus impinging on the underlying A1 vessel (Figure 3A). The aneurysmal dome was dissected circumferentially and then the RAH was clipreconstructed using two side-opening Yasargil clips that were placed parallel to the vessel, along the long axis of the aneurysm (Figure 3B; Video). Unfortunately, intraoperative angiography demonstrated that both the aneurysm and the RAH were completely obliterated by the clip (Figure 1G). However, intraoperative

From the Department of Neurosurgery, University of Louisville, Louisville, KY, USA (KM, RR); Department of Neurosciences, Virginia Commonwealth University School of Medicine - Inova Campus, Falls Church, VA, USA (RR).

Received February 1, 2015. Final Revisions Submitted June 17, 2015

Correspondence to: Ralph Rahme, Inova Neuroscience Institute, 3300 Gallows Rd, Falls Church, VA 22042. Email: rrahme@waln.org 

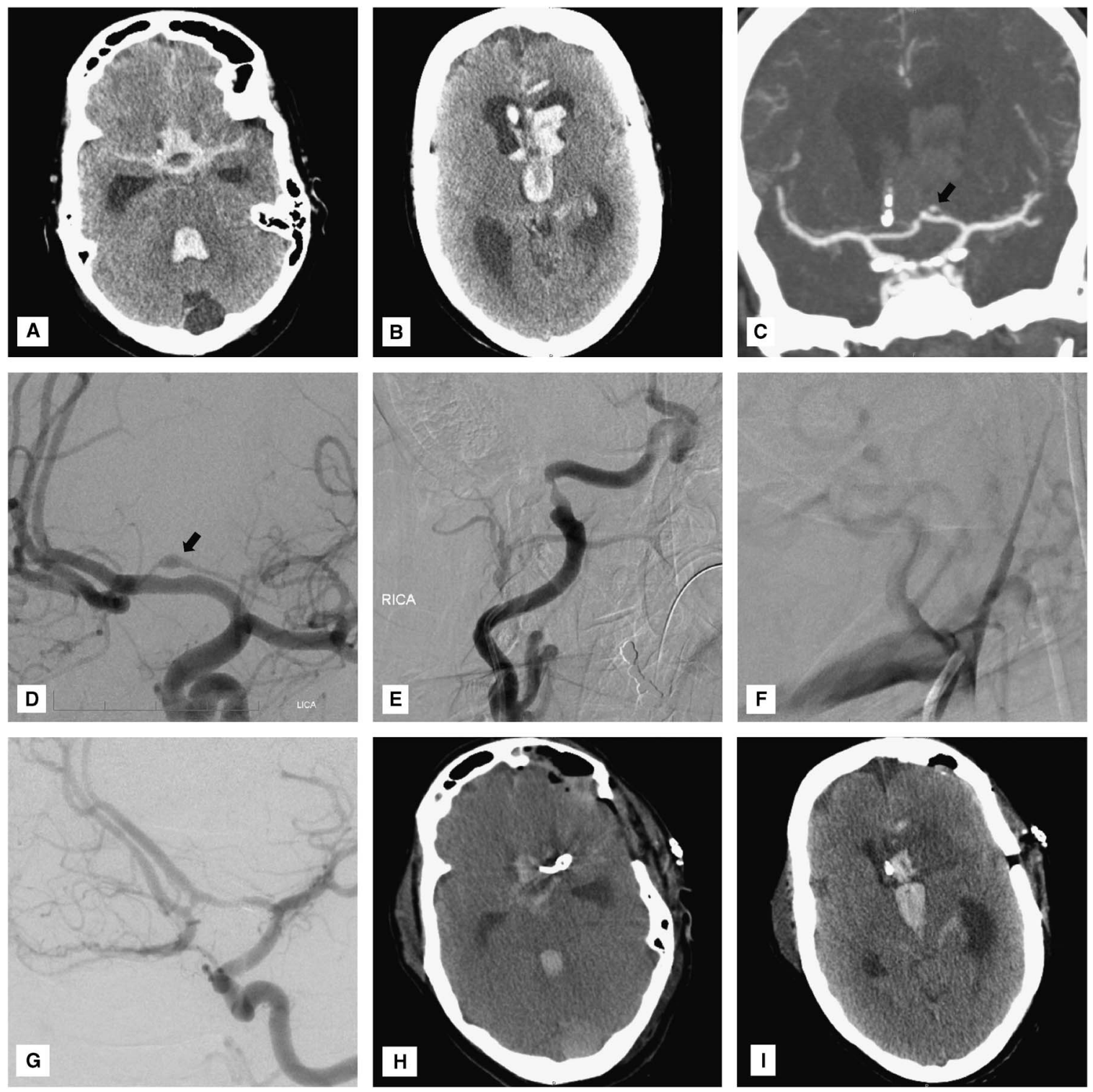

Figure 1: $(A, B)$ Head CT reveals massive cisternal subarachnoid and intraventricular hemorrhage with hydrocephalus. (C) $C T$ and $(D)$ catheter angiography show a small fusiform aneurysm arising from the proximal portion of the left recurrent artery of Heubner (arrows). Note the deformation of the superior wall of the underlying Al vessel. Cerebral angiography reveals evidence of prior dissections of the $(E)$ cervical right internal carotid and $(F)$ right vertebral arteries. $(G)$ Intraoperative angiography demonstrates complete obliteration of both the aneurysm and the left recurrent artery of Heubner. (H, I), Postoperative head CT shows an expected small infarct limited to the head of the left caudate nucleus.

motor-evoked potentials remained normal, suggesting that a left internal capsular infarct was unlikely to occur despite occlusion of the RAH. Thus, the aneurysm clips were left in place.

Postoperatively, the patient's neurological condition was unchanged from baseline, with persistence of a symmetric motor examination (withdrawal of all four extremities to pain). Head CT revealed an expected small infarct in the head of the left caudate nucleus, without involvement of the internal capsule (Figure 1H,I). Unfortunately, 3 days later (SAH day 6), the patient developed severe diffuse cerebral vasospasm (Figure 4A-C) with marked neurological deterioration to a Glasgow coma score of 3T. Despite maximal medical treatment (triple $\mathrm{H}$ therapy) and intra-arterial verapamil infusion, she developed extensive bihemispheric infarctions (Figure 4D-F). Given her poor baseline and current neurological condition, and the severity and refractoriness of her vasospasm, the family requested to withdraw medical care, and the patient ultimately died.

\section{DISCUSSION}

To the best of our knowledge, this is the first reported case of a dissecting aneurysm of the RAH and only the second report of a dissecting aneurysm occurring in a patient with OI. Although a case of dissecting aneurysm extending from the ACA into the RAH has been previously reported in a non-OI patient, ${ }^{6}$ that case, in contrast to the present report, does not represent a pure RAH dissection. 



Figure 2: Photographs of the patient's daughter demonstrate typical clinical features of osteogenesis imperfecta: $(A, B)$ blue-tinted sclerae and $(C, D)$ joint hyperlaxity.
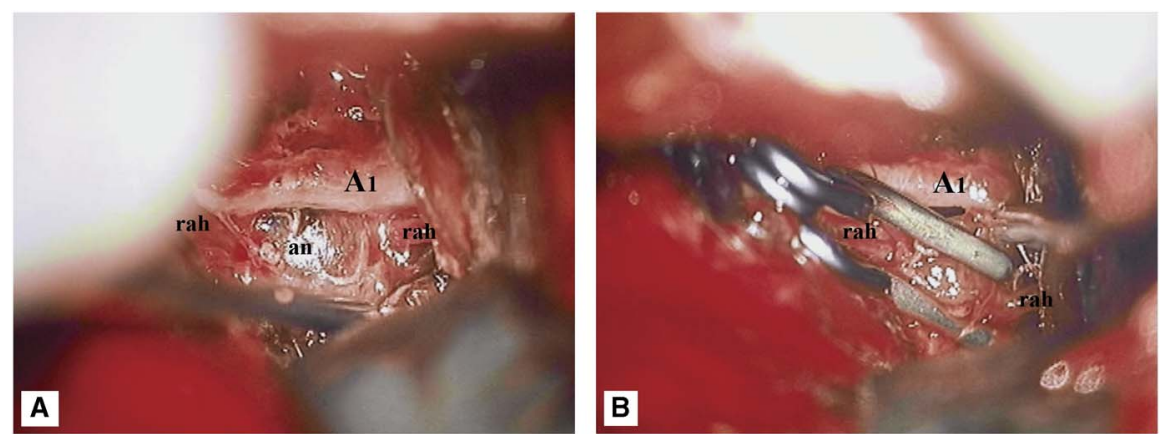

Figure 3: Intraoperative microphotographs demonstrating the dissecting aneurysm (an) of the left recurrent artery of Heubner (rah). (A) Note the large intramural thrombus impinging on the underlying Al vessel. (B) Attempted clip reconstruction of the recurrent artery of Heubner, using two side-opening Yasargil clips.

\section{RAH aneurysms}

The RAH is a large perforator that arises from the ACA in the region of the anterior communicating artery, usually from the proximal A2 segment or, less commonly, from the distal A1 segment. It doubles back on the A1, hence the term "recurrent," and travels between it and the frontal lobe until it reaches the region of the carotid bifurcation, where it enters the brain through the anterior perforated substance. The mean diameter of the RAH is $1 \mathrm{~mm}$, but may vary from 0.2 to $2.9 \mathrm{~mm} .{ }^{5}$ Aneurysms of the RAH have not been previously reported. The RAH supplies blood flow to the anterior part of the caudate nucleus, putamen, and globus pallidus externus; the anteroinferior portion of the anterior limb of the internal capsule; the uncinate fasciculus; and the anterior hypothalamus. Lesions to this artery may result in a range of neurological deficits, including contralateral brachiofacial hemiparesis, aphasia (if dominant hemisphere), and even memory or personality changes. ${ }^{5}$ Fortunately, in many cases, occlusion of this artery leads to clinically silent infarctions that are limited to the head of the caudate nucleus, as was the case in the present patient.

\section{Intracranial dissections}

The management of intracranial dissections remains controversial. Although many cases in the literature have been successfully managed conservatively and have healed spontaneously, early securement of ruptured dissecting aneurysms is generally 
advocated, given the high risk of early rerupture. ${ }^{1-4}$ Thus, although conservative management in this patient would have been a potential treatment option, neither the treating team nor the patient's family felt comfortable leaving that ruptured dissecting aneurysm unsecured. Unlike saccular aneurysms, dissecting aneurysms are challenging to treat by simple clip ligation or coil embolization, given the lack of a well-defined neck and actual involvement of the parent vessel wall. As such, definitive treatment usually involves surgical or endovascular trapping or proximal occlusion, with or without extracranial-intracranial bypass. ${ }^{1,2}$ In fact, even when an attempt was made to clip-reconstruct the dissected RAH in this patient, our efforts to preserve the vessel were ultimately unsuccessful. This outcome is not very surprising, given the very small caliber of the RAH and the dissecting fusiform nature of the aneurysm. Although techniques aimed at preserving flow in dissected vessels, such as surgical wrapping or clip-wrapping and endovascular stenting, have been advocated by some, ${ }^{1}$ the efficacy of such techniques in preventing aneurysm rerupture remains unproven. Thus, whenever possible, we advocate complete exclusion of the aneurysm from the circulation. Had intraoperative motorevoked potentials deteriorated following clip occlusion of the RAH in this patient, then consideration would have been given to these alternative flow-preserving treatment options.

\section{Osteogenesis imperfecta}

OI or brittle bone disease is a group of inherited connective tissue disorders characterized by a deficient synthesis of collagen type I. There are at least eight recognized types of OI, with type I being the mildest and most common. OI is most commonly caused by mutations to the COL1A1 (chromosome 17q) or COL1A2 (chromosome 7q) gene, which follow autosomal dominant transmission with variable expression. However, up to one-third of cases may be sporadic. ${ }^{7-9}$ Although diagnosis of OI is primarily clinical, genetic testing can help confirm the diagnosis in cases of uncertainty. In the present case, both the patient's brother and daughter had confirmed COL1A2 gene mutation.

The clinical features of OI vary greatly from person to person, even among people with the same type and within the same family. Although the most severe forms may result in death at birth or during infancy, the mildest forms can be associated with a normal life expectancy. Skeletal pathology, including fragile bones and frequent pathologic fractures, is a hallmark of the disease. Likewise, blue sclerae are a characteristic finding and help distinguish OI from other heritable connective tissue disorders. Other manifestations may include fragile skin, easy bruising, muscle weakness, joint laxity, curved bones, short stature, scoliosis, brittle teeth (dentinogenesis imperfecta), hearing loss, and restrictive pulmonary disease..$^{7-9}$

\section{OI and cerebrovascular pathology}

Compared with other connective tissue disorders, OI has been less commonly associated with cerebrovascular pathology. In fact, there is only a handful of case reports describing intracranial aneurysms in patients with OI, with the majority being saccular in
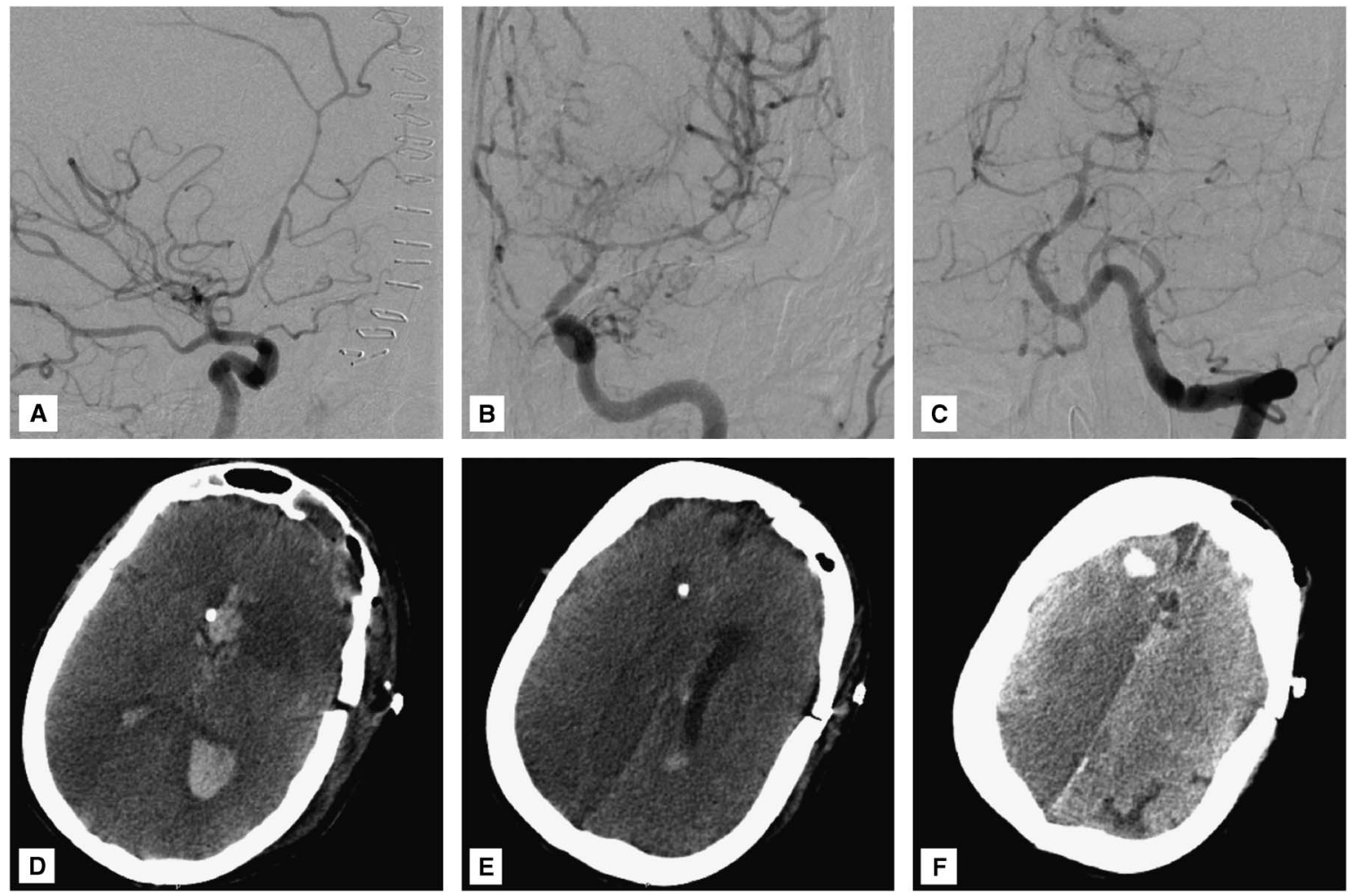

Figure 4: (A) Right internal carotid, (B) left internal carotid, and $(C)$ left vertebral angiograms demonstrate severe diffuse (proximal and distal) vasospasm involving the bilateral anterior cerebral arteries, left middle cerebral artery, and vertebrobasilar circulation. (D-F) Head CT reveals multiple bihemispheric infarctions secondary to vasospasm.

Video: Microsurgical clipping of a dissecting aneurysm of the left recurrent artery of Heubner. 
nature, arising from typical locations, including the anterior communicating, basilar, or middle cerebral artery. ${ }^{10}$ Only one previous report exists of a ruptured intracranial dissecting aneurysm in the setting of OI, which involved the superior cerebellar artery. ${ }^{11}$ Interestingly, the patient described here also had evidence of prior extracranial dissections of the right internal carotid and vertebral arteries, which likely reflects underlying vascular wall fragility secondary to OI.

\section{ConClusions}

Although rare, intracranial dissections can occur in the setting of OI and can lead to devastating intracranial hemorrhage. Dissecting aneurysms in patients with OI may occur in atypical locations and have a predilection for smaller and more distal arteries, such as the RAH. Management of such aneurysms is particularly challenging and preservation of the parent vessel is often impossible. Intraoperative angiography and neurophysiological monitoring can provide very useful feedback in this setting.

\section{DisClosures}

The authors have nothing to disclose.

\section{REFERENCES}

1. Uhl E, Schmid-Elsaesser R, Steiger HJ. Ruptured intracranial dissecting aneurysms: management considerations with a focus on surgical and endovascular techniques to preserve arterial continuity. Acta Neurochir (Wien). 2003;145:1073-84.

2. Yonekawa Y, Zumofen D, Imhof HG, Roth P, Khan N. Hemorrhagic cerebral dissecting aneurysms: surgical treatments and results. Acta Neurochir Suppl. 2008;103:61-9.

3. Mizutani T. Natural course of intracranial arterial dissections. J Neurosurg. 2011;114:1037-44.

4. Ono H, Nakatomi H, Tsutsumi K, et al. Symptomatic recurrence of intracranial arterial dissections: follow-up study of 143 consecutive cases and pathological investigation. Stroke. 2013;44:126-31.

5. Rhoton AL Jr. The supratentorial arteries. Neurosurgery. 2002; 51(4 Suppl):S53-120.

6. Pilz P. Dissecting aneurysm of the anterior cerebral artery and Guillain-Barré-syndrome during pregnancy [in German]. J Neurol. 1977;214:295-9.

7. Rauch F, Glorieux FH. Osteogenesis imperfecta. Lancet. 2004;363:1377-85.

8. Khandanpour N, Connolly DJ, Raghavan A, Griffiths PD, Hoggard N. Craniospinal abnormalities and neurologic complications of osteogenesis imperfecta: imaging overview. Radiographics. 2012; 32:2101-12

9. Osteogenesis Imperfecta Foundation. Facts about osteogenesis imperfecta. Available from: http://www.oif.org/site/PageServer? pagename=AOI_Facts [Accessed October 6, 2014].

10. Hirohata T, Miyawaki S, Mizutani A, et al. Subarachnoid hemorrhage secondary to a ruptured middle cerebral aneurysm in a patient with osteogenesis imperfecta: a case report. BMC Neurol. 2014;14:150.

11. Matouk CC, Hanbidge A, Mandell DM, Terbrugge KG, Agid R. Osteogenesis imperfecta, multiple intra-abdominal arterial dissections and a ruptured dissecting-type intracranial aneurysm. Interv Neuroradiol. 2011;17:371-5. 